Témoigner Témoigner. Entre histoire et mémoire

Getuigen Revue pluridisciplinaire de la Fondation Auschwitz

$116 \mid 2013$

Voyages mémoriels

\title{
Dire et écrire le massacre dans « Ayataki » de Sócrates Zuzunaga Huaita
}

Speaking and writing about the massacre: "Ayataki" by Sócrates Zuzunaga

Huaita

Spreken en schrijven over de massamoord. 'Ayataki' van Sócrates Zuzunaga

Huaita

\section{Mylène Herry}

\section{(2) OpenEdition}

Journals

Édition électronique

URL : http://journals.openedition.org/temoigner/363

DOI : 10.4000/temoigner.363

ISSN : 2506-6390

Éditeur :

Éditions du Centre d'études et de documentation Mémoire d'Auschwitz, Éditions Kimé

Édition imprimée

Date de publication : 1 septembre 2013

Pagination : 91-99

ISBN : 978-2-84174-643-9

ISSN : 2031-4183

\section{Référence électronique}

Mylène Herry, « Dire et écrire le massacre dans « Ayataki » de Sócrates Zuzunaga Huaita », Témoigner. Entre histoire et mémoire [En ligne], 116 | 2013, mis en ligne le 01 juin 2015, consulté le 23 octobre 2020. URL : http://journals.openedition.org/temoigner/363 ; DOI : https://doi.org/10.4000/temoigner. 363 


\title{
Dire et écrire le massacre dans Ayataki' de Sócrates Zuzunaga Huaita
}

\section{MYLĖNE HERRY}

Framespa UMR 5136 (CNRS), Toulouse Le Mirail II

\begin{abstract}
Je voudrais que mes livres aident les gens à devenir meilleurs, à mieux comprendre le monde dans lequel j’ai vécu et à purifier leur âme ; que la lecture de mes livres éveille l'amour chez les hommes, le désir de lutter pour les idéaux d'humanisme et de progrès de nos peuples oubliés jusqu'à maintenant ${ }^{1}$.
\end{abstract}

crit en 1988 par le Péruvien ayacuchanais, Sócrates Zuzunaga Huaita, Ayataki est le récit bref d'un paysan andin, témoin et victime de la violence qui secoue alors les Andes. À travers un monologue continu dépourvu de points, le narrateur - Timoteo - raconte à « Mamacita » le massacre commis dans le village : suspectant les habitants du village d'appartenir au groupe terroriste, le Sentier Lumineux, les militaires les tuent. En réalité, les membres du Parti $^{2}$ ne sont pas là. Quand Cornelio, le capitaine de l'armée péruvienne, arrive, il se rend compte qu'une des victimes est le professeur du village et qui se trouve être son propre frère, Modesto.

Rappelons qu'au moment de l'écriture de la nouvelle, le Pérou endure une guerre interne extrêmement violente depuis la proclamation en mai 1980 de la lutte armée par le Sentier Lumineux. Pour rétablir l'ordre, une militarisation des Andes s'organise dès 1982. En fin de compte, la présence des militaires ne fera que renchérir l'intensité du conflit dans l'imposition de la loi du plus fort. Dans ce théâtre de l'horreur, la population andine est cerclée de part et d'autre. Exposée en première ligne, elle subit les dommages d'une violence généralisée et arbitraire. En 1988, les textes traitant

(1) Sócrates Zuzunaga Huaita, « Ayataki », in Cuento peruano en los años de violencia, selección por Mark R.Cox, Lima, Editorial San Marcos, 2000, p. 183-188.

(1) Extrait de l'entretien de Sócrates Zuzunaga Huaita du 30 mai 2009, “Maestro, narrador y músico”. Document consulté on-line le 20/01/2010 : http://www.pauzasur.com/index.php?option=com_content\&view=article\&id=53: entrevista-a-socrates-zuzunaga-\&catid=37:s-zuzunaga

(2) Le Sentier Lumineux s'appelle le Parti Communiste Péruvien - Sentier Lumineux (PCP-SL). 
la thématique de la violence politique sont peu nombreux, en partie parce que le conflit représente une réalité lointaine, souvent ignorée et/ou peu relayée par la « ciudad letrada $»^{3}$. Alors, la transcription du témoignage de Timoteo, depuis une plume ayacuchanaise ${ }^{4}$, permet de visualiser et d'appréhender la guerre interne depuis un nouveau point de vue, celui du subalterne.

Je propose donc de m'interroger sur la manière dont le conflit péruvien des années 1980 est raconté et mis en scène dans l'épisode en question. Je m’intéresserai pour cela au traitement du contrat social propre aux communautés représentées et à la relation à l'Autre dans sa considération et sa reconnaissance. Enfin, j’étudierai comment le langage reflète les crises supportées.

\section{UNE SOCIÉTÉ PÉRUVIENNE DÉCHIRÉE}

Dans le Pérou de l'année 1988, la lutte armée - initiée par le Parti Communiste Péruvien - Sentier Lumineux (PCP-SL) - est engagée depuis huit ans, suite à douze ans d'instabilité gouvernementale. Les tactiques des sentiéristes, menés par le professeur Abimael Gúzman, consistaient à assassiner les représentants des autorités de la zone intérieure pour créer un espace vide et imposer ses propres instances et son idéologie. Lanthropologue Carlos Iván Degregori définit sa stratégie :

Dans son projet et son action, le marxisme-léninisme-maoïsme, parti central et scientiste, va dans le sens de la recherche du progrès, mais aussi et surtout revêt les aspects les plus autoritaires, fermés, exclusifs et prémodernes de la culture politique péruvienne. Ces aspects sont exacerbés dans l'Ayacucho des années 1960 et 1970, mais ne sont pas propres à cette région ; ils imprègnent, à des degrés divers, une bonne partie du pays et créent les conditions de l'expansion du PCP-SL au-delà de son espace géographique et social originel $^{5}$.

Lorganisation terroriste a déployé une violence dirigée contre les ennemis du peuple, dirigeants populaires et autorités traditionnelles, pour former une république populaire de nouvelle démocratie. La naissance de cette violence politique fut l'expression d'une crise sociale profonde. La construction d'une république «sans l'Indien et même contre l'Indien » - si nous considérons les textes de José Carlos Mariátegui - condense et articule les structures socio-culturelles de la domination coloniale. Face à la hiérarchie des classes et des races, la fracture nationale s'ouvre et les conflits sociaux s'organisent. Létat oligarchique péruvien (loin de la réalité andine et de ses préoccupations) tend à mépriser le peuple andin en l'ignorant ou

(3) Titre de l'œuvre de Ángel Rama - La ciudad letrada, Hanover (U.S.A.), Ediciones del Norte, 2002 [1984] - qui désigne à l'évidence la relation du pouvoir et du monde intellectuel.

(4) Ayacucho est la région andine ; elle est considérée comme le berceau du Sentier Lumineux et la zone rouge du conflit.

(5) Carlos Iván Degregori, Ayacucho 1969-1979. El nacimiento de Sendero Luminoso, Lima, Instituto de Estudios Peruanos ediciones, 1990, p. 212. 
en refusant de considérer sa pauvreté. Pendant le conflit interne (1980-2000), les militaires, en tant que représentants des intérêts de l'oligarchie, pénètrent dans le territoire de l'Autre, souvent méconnu. Alors, pour eux, qui sont les subversifs? Depuis un imaginaire liménien, ne seraient-ce pas les Indiens, les Autres, anonymes et différents ? Cependant, comment les différencier des comuneros, témoins et victimes, qui demandent de l'aide? Dans cette confusion, comment faire confiance à l'Autre et ne pas devenir paranoïaque?

À travers le monologue de Timoteo, il suffit d'utiliser les termes terrukos et milikos pour contextualiser la narration. Mais, tout au long du témoignage, nous nous rendons compte que les terrukos ne sont pas représentés et n'agissent pas dans l'anecdote racontée. Ce sont les militaires qui, pour provoquer un sentiment de terreur, utilisent ce terme à travers l'intrusion d'interjections. Recourir au discours direct des bourreaux qui instrumentalisent le mot terrukos permet à Timoteo de ne pas l'assumer. On distingue dans l'exemple suivant le double niveau d'énonciation: «[...] desde ahí vi cómo también a nuestro profe lo golpeaban y pateaban en el suelo, terruko conchetumadre, comunista de mierda, ahora vas a ver hijueputa [...]» (p. 184).

L'Andin Timoteo est le témoin du massacre. Le témoignage identifie clairement les coupables, los milikos : «[...] así me metí en una choza deshabitada y me subí al techo, como una lagartija asoleándose, y así pude ver a todos los uniformados, con sus caras negras con pasamontañas [...]» (p. 185).

La terreur se généralise malgré l'absence des sentiéristes. Bien que les villageois tentent de convaincre les militaires de la confusion, ces derniers ont un seul objectif: trouver les coupables, ceux qu'ils auront désignés, les tuer. La loi du plus fort nie l'évidence. La nation andine explose :

[...] así llegaron a nuestra casita, abran la puerta carajo, rápido concha de sus madres, y como nos tardamos en abrir, „pajraraj!, pobre mi puertita al suelo, y dónde está ese hijueputa, dónde está ese mierda, pero qué hijueputa, qué mierda, nosotros no sabemos nada papacitos, cómo que no saben nada jijunagramputas, toma toma toma, para que aprendas a no mentir, para que aprendas a decir la verdad, pobre mi lomito charqueado quedó con los golpes, ayayau ayayaucito [...] (p. 184).

Toutes les références renvoient aux croyances, à la nature et au peuple andin. Par exemple, nous trouvons les huaicos et le serpent Amaru, qui, au début du conte, annoncent la tragédie : « Llegaron como a la medianoche, mamacita, haciendo una bulla tremenda, como cuando llega un huaico hartazo por la quebrada, como cuando el Amaru se sacude de la tierra [...] » (p. 183). La violence s'exprime à travers des comparaisons et des métaphores appartenant à la culture andine. Ainsi, la voix des milikos résonne comme « trueno de lluvia [...]» (p. 186) et les balles retentissent « [...] como maíz tostándose en la kallana [...]» (p. 186).

Mais c'est le mélange des langues, le castillan avec le quechua, qui affirme une volonté de réalisme. 
[...] nos quedamos llorando y llorando, harto asustados, pellizcándonos el sentimiento con muchas preguntas malagüeras, diciendo para nuestro adentro, adónde se lo llevaran a nuestro profe, pobrecito, qari wawa, tan bueno y cariñoso con nuestros niños, ayayay [...] (p. 185).

La nouvelle aborde donc le conflit du point de vue andin, conflit perçu, en partie, à travers une réactivation de la réalité ancestrale et le rapport à la nature. Selon Sócrates Zuzunaga Huaita, il y a là une des caractéristiques des auteurs de la sierra qui permet de les distinguer des écrivains de la costa qu'il considère, dans leur rejet de l'environnement, « amputés » et incomplets :

Nous, les écrivains de la «sierra», nous chantons la nature andine, sabeauté incomparable, ses vents, son froid rigoureux, la caresse de sa pluie... Tout cela un écrivain de la côte ne peut le ressentir. C'est la raison pour laquelle ils ne nous comprennent pas et nous marginalisent ; ils sont sans âme ; ils sont vides, creux à l'intérieur ${ }^{6} . .$.

\section{RUPTURE DU CONTRAT SOCIAL}

À travers le témoignage du narrateur, nous pouvons identifier la communauté dans ses mœurs socioculturelles et sa relation à l'Autre. Avant tout, il faut remarquer la tolérance et le respect du peuple qui ne résiste pas au traitement des bourreaux. Les militaires refusent de communiquer avec l'Autre et assoient leur autorité et leur supériorité par la terreur qu'ils provoquent dans la famille de Timoteo. Ils ne parlent pas mais crient ; ils insultent et frappent. Le narrateur dit: « [...] me gritaron, [...] cierra la boca carajo, indio conchetumadre, y jua y jua y jua, me revolcaron a culatazos en el suelo [...] » (p. 184). En arrivant au village, les militaires détruisent l'ordre établi et l'autorité, représentée par Modesto, l'instituteur. Ils s'imposent par la violence et ne respectent plus personne.

On remarque avec évidence tout au long du conte des métaphores antagoniques qui tendent à caractériser, d'une part, les actes robotiques des uns et, d'autre part, les actes humains des autres. En effet, l'humanisme des Andins s'oppose à la déshumanisation des milikos. Le témoignage du comunero - depuis un point de vue interne - permet au lecteur d'être en empathie avec les victimes et de partager les plaintes, les prières, la douleur et l'incompréhension.

[...] y yo llorando con toditas mis lágrimas, con mi boca destrozada y mis labios partidos y mi lengua rajada, rogaba a todos los Taitachas del cielo para que no lo encuentren papay, qué ojala nuestro buen profe se haya escondido bien [...] (p. 184).

[...] que no podíamos sujetar nuestro llanto, todos arrodillados en el suelo, temblando como

(6) Extrait de l'entretien de Sócrates Zuzunaga Huaita du 30 mai 2009, “Maestro, narrador y músico". Document consulté on-line le 20/01/2010 : http://www.pauzasur.com/index.php?option=com content\&view=article\&id=53: entrevista-a-socrates-zuzunaga-\&catid=37:s-zuzunaga 
cuerdas de arpa, como telarañas al viento, y mi Sebastiana, mamay, en mi lado, lloraba como un loca, jaloneándome de los pelos, punteándose el pecho, a veces empujándome, otras veces rogándome [...] (p. 185).

À la sensibilité du peuple s'oppose le sadisme de l'uniforme, anonyme. Timoteo raconte:

[...] gritaban con voz de chanchón renegón, no se muevan carajo, al que levante la cabeza le perforo los sesos, y disparaban por encima de los echados, pam pam pam, y seguían gritando con sus voces roncas [...] y les pateaban en las costillas, les pisaban en la cabeza, ahora ya se jodieron hijos de la gramputa, y en eso nomás, a la señal de uno de los milikos, ordenaron con voz más fuerte, como trueno de lluvia, ahora váyanse mierdas, levántense y corran, escapen o aquí se mueren todos, y empezaron a dispararles muy cerquita del cuerpo, levantando polvo de muy cerca de sus cabezas [...] (p. 186).

Face à cette déshumanisation des militaires, les victimes, parmi lesquelles Modesto - « un hombre bien plantado que no se dejaba pisar el poncho con nadie » (p. 187) -, perdent leur dignité : elles s'animalisent («vizcachas asustadas » p. 186) et cherchent leur mère et leurs dieux pour sauver leur vie. Ils connaissent leur destin et attendent déjà como muertos leur malheur. Témoin oculaire, Timoteo continue de témoigner :

[...] y los pobrecitos empezaron a caer como ramas macheteadas, gritando desesperadamente, llamando a sus mamacitas, llamando a sus hijitos, clamando a Diosito lindo, con las bocas masticando el aire, arañando el suelo, y así se quedaron tiesos, silenciosos, regando con su sangre el polvo de nuestra plaza, como perros ordinarios [...] (p. 186).

Modesto est pour les militaires « un animal raro » (p. 186). Au-delà de son rôle éducatif, « el buen hombre », fut un défenseur de la justice et des abus des « malas autoridades » (p. 187). Le choix de son prénom, dans ce sens, est significatif et induit une critique de la prétention militaire. De la même manière, nous nous rendons compte de l'importance de l'onomastique des comuneros. Ainsi, Timoteo, Timao Theos en grec, est celui qui honore les dieux. Il croit en la justice et tend à protéger les siens. Son rôle devient presque apostolique si nous considérons la métaphore utilisée pour caractériser les ennemis de Dieu, « esos satamanes »(terme répété deux fois, p. 185, 186) dont l'orgueil est synonyme de prétention extrême. Le monde devient le royaume de l'enfer et de la terreur. Selon le texte, les démons sont des bêtes semblables aux loups qui chassent leur proie sans défense. «[...] y pateando a todas las puertas, metiendo el hocico hasta debajo de las camas, sin respetar a nadie, ni a Cristo, ni a rey, todas fieras rabiosas, gritoneando como unos mismos condenados [...]» (p. 183).

Dans le récit bref, la conscience s'oppose à l'inconscience, les valeurs authentiques aux fausses, et l'éducation à l'endoctrinement. La communauté veut s'ouvrir à l'Autre 
au moyen du système éducatif. D’ailleurs, Cornelio et Modesto, les frères, sont partis sur la côte pour étudier, et ce fut grâce à ce séjour qu'ils obtinrent une reconnaissance sociale. Voici comment Timoteo témoigne du parcours de Modesto :

[...] y fue así que el Modesto regresó de la costa hecho todo un señor profesor, masticando un buen castellano, con buena iluminación en la cabeza, y se puso a enseñar en nuestra escuelita, haciéndose querer por todos nosotros porque bien bonito enseñaba a nuestros maqtillos, sin jalarles las orejas, sin tirarles látigo, con harto cariño y regalitos mas bien [...] (p. 187).

Pourtant, paradoxalement, l'éducation devient, dans le texte, le motif du massacre. En effet, les militaires, à cette époque-là, connaissent le mode de propagande de l'idéologie maoïste, c'est-à-dire la création des écoles populaires dans lesquelles on enseigne les textes fondamentaux de la révolution de masse. C'est par ce moyen que les sentiéristes se sont infiltrés dans les populations pour recruter des soldats. Cet amalgame entre les professeurs actifs dans ce processus propagandiste et ceux qui continuent à éduquer les « maqtas » (p. 185) selon des principes républicains permet en quelque sorte de légitimer le comportement criminel adopté par les forces armées. Dans le doute, ils ne firent aucune différence entre les uns et les autres. L'autorité nationale, menée ici par Cornelio, ne sait que recourir à la violence physique et verbale pour s'imposer. Par opposition, Modesto représente l'autorité locale, celle qui n'a pas besoin de violenter pour transmettre aux élèves et répond donc à l'ordre andin dont j’ai parlé.

L'anecdote andine devient universelle, tout d'abord à travers la connotation biblique du premier fratricide de l'Histoire, le meurtre d'Abel par Caïn, respectivement, dans le conte, de Modesto par Cornelio. De fait, « el rebaño de ovejas » fait directement référence à la scène biblique. La mort de Modesto est une punition des crimes de guerre du commandant Cornelio. Toute l'histoire humaine semble répondre à ces deux figures pour des raisons opposées : d'une part, la foi et la tendresse de cœur ; d'autre part, la fierté et la violence. Depuis lors, ces antagonismes régissent les relations humaines, transcendées dans un contexte de guerre civile. Aussi, il est à relever la connotation musicale que propose le conte. En effet, au-delà de signifier Ayataki, la douleur, le mot quechua fait référence à un chant pour les défunts. Cette forme du dire se transcrit dans le texte à travers un langage qui bouleverse les règles narratives établies.

\section{UN NOUVEAU PACTE DE LECTURE ?}

Rappelons que le narrateur, Timoteo, est en train de parler à Mamacita, la mère de Modesto et Cornelio. Malgré son âge et la distance, il s'est précipité chez «l'ancienne » pour lui raconter le massacre et lui annoncer la mort de son fils. Ce témoignage confidentiel se caractérise par une écriture fragmentée qui traduit la précipitation et la panique de Timoteo par le souvenir de l'expérience. Il nous invite à découvrir et à 
partager la réalité de son monde, ce qui, pour l'auteur, définit la fonction principale de la littérature :

Pour moi, la littérature est avant tout la chronique spirituelle et matérielle d'un peuple... Je vois continuellement comment vivent les paysans de mon village. Et, plongé dans ce monde où il m’a été donné de naître et de vivre, je sens en moi un devoir intime qui me pousse à écrire sur cette vie-là?.

Il serait donc légitime de se demander comment la production narrative propose d'exemplifier la crise de la société péruvienne.

Tout d'abord, il faut dire que le texte n'est qu'un extrait du message oral puisqu'il s'ouvre et se ferme par des points de suspension et ne présente aucune majuscule. La ponctuation a, ici, une fonction rythmique évidente. Le meilleur exemple est l'absence de points à la fin des phrases. Le lecteur ne peut pas s'arrêter et, malgré les nombreuses virgules, la lecture lui coupe la respiration. En effet, ces dernières permettent l'alternance des discours : le monologue à la première personne se confond avec le discours direct relatif à l'anecdote. Dans une théâtralisation de l'expérience, le lecteur attribue à chacun le lexique qui lui correspond et identifie les voix. Ainsi, les milikos sont les barbares qui utilisent un vocabulaire irrespectueux comme « jijunagramputas, hijueputas, conchatumadre... » alors que les Andins, peureux, implorent : « [...] por favorcito, tenga compasión de nosotros, no nos hagan daño, no nos maten, tenemos hartos hijitos que mantener [...] (p. 184).

Le texte devient " cette machine cybernétique ${ }^{8}$ » propre au genre théâtral ; il donne au lecteur des informations multiples concernant l'expérience, le décor et les personnages de façon simultanée, mais à des rythmes différents. Par exemple, nous pouvons apprécier trois espaces énonciatifs dans cet extrait :

[...] y en ahí no nomás, mamacita linda, todos nuestros muchachitos se levantaron y empezaron a correr como vizcachas asustadas, a la gana gana, para donde sea, unos hacia abajo, otros hacia arriba, sin saber ni presentir lo que estaba esperando, y en eso las balas empezaron a reventar como maíz tostándose en la kallana, pam pam pam pam [...] (p.186).

Le narrateur passe par trois niveaux d'écriture : d'abord, comme toile de fond, le récit descriptif de l'épisode passé, narré par Timoteo ; puis les indices du présent énonciatif, celui qui correspond à l'interjection soulignée, « Mamacita linda », destinée à son auditrice ; et, enfin, le temps de l'anecdote - présenté, ici, en caractères gras - qui met en scène les principaux acteurs dans le discours direct ou qui, par des onomatopées, nous plonge dans l'atmosphère scénique. Ces changements discursifs

(7) Extrait de l'entretien de Sócrates Zuzunaga Huaita du 30 mai 2009, “Maestro, narrador y músico”. Document consulté on-line le 20/01/2010 : http://www.pauzasur.com/index.php?option=com_content\&view=article\&id=53: entrevista-a-socrates-zuzunaga-\&catid=37:s-zuzunaga

(8) Roland Barthes, «Littérature et signification » in Essais critiques, Paris, Seuil, 1981 [1963], p. 258. 
génèrent donc d'autres changements de nature spatiale, émotionnelle et intonative. Dans ce sens, il faut souligner la crise des genres littéraires dont les frontières ont fortement été déstabilisées. Ce récit bref, inclus dans une anthologie de contes, ne définirait-il pas en réalité un monologue de théâtre poétisé par un langage ponctué d'images propres à l'ordre andin?

Il faudrait maintenant préciser la présence fondamentale du lyrisme dans ce conte. En effet, le sujet lyrique s’énonce dans une double référence : le « je » et le monde dans le fait de caractériser à la fois l'expression des sentiments personnels et le caractère universel du thème traité. Notons que Sócrates Zuzunaga Huaita est aussi musicien et ne peut concevoir la littérature sans l'art. Ce qu'il exprime ainsi :

Les liens de l'art et de la littérature avec la vie du peuple et avec la nature sont inaltérables. Quand j'écoute un « huaino » ou quand je lis un conte au thème andin, se présentent à mon esprit des images très familières et attendrissantes. Je perçois mon peuple, ses montagnes, ses bois, ses sources, ses rivières, son soleil, sa pluie, ses soirs de pleine lune... Et tout, tout, m'évoque les notes plaintives d'un « charango » ou la lamentation solitaire d'une « quena » qui gémit au loin... Dans les paroles andines d'un « huaino » ou dans le monde fascinant d'un conte situé dans le Pérou profond se reflètent nombre de chemins caillouteux et épineux, chemins accidentés avec leurs côtes et leurs pentes, sur lesquels s'affaire notre peuple montagnard. Dans notre littérature comme dans celle des autres peuples andins s'expriment aussi bien les sujets sociaux que les sentiments lyriques de l'homme, son univers spirituel et ses aspirations'.

La poésie se rapproche donc de la musique dans la création d'un langage spécifique qui accorde le son et le contenu, l'implicite et le dit. En effet, tout au long du conte, le traitement du langage traduit une oralité. Par exemple, les polysyndètes, les onomatopées et les anaphores suggèrent, à travers un rythme ternaire, d'une part, le massacre - «[...] y disparaban por encima de los echados, pam pam pam [...] » (p. 186) - et, d'autre part, la violence physique : « [...] y jua jua jua, me revolcaron a culetazos [...] ( (p. 184). La répétition systématique de sons fait référence à la volonté de donner du rythme à la production orale et marque aussi la cruauté des militaires. En revanche, la répétition de la voyelle « $\mathrm{i}$ » dans l'adjectif « triste » traduit les pleurs du narrateur et renoue avec le lyrisme précédemment évoqué : «[...] con una tonada de ayataki, triiiiiiiste, bien triste [...]» (p. 185).

Grâce au maniement minutieux du langage de Sócrates Zuzunaga Huaita, le texte devient poétique. Les fragments entre virgules s'associent aux vers poétiques. Le choix de s'exprimer au moyen d'images et de métaphores est caractéristique du genre poétique, ce qui formalise encore plus l'écriture :

(9) Extrait de l'entretien de Sócrates Zuzunaga Huaita du 30 mai 2009, “Maestro, narrador y músico". Document consulté on-line le 20/01/2010 : http://www.pauzasur.com/index.php?option=com content\&view=article\&id=53: entrevista-a-socrates-zuzunaga-\&catid=37:s-zuzunaga 
[...] y eso nomás el pueblo se volvió silencioso,

como silencio de muerte,

y algunos perros nomás estaban aullando en la lejanía,

y los eucaliptos también lloraban con el viento de la madrugada,

con una tonada de ayataki,

triiiiiiiste, bien triste,

y, nosotros que no podíamos sujetar el llanto,

todos,

arrodillados en el suelo,

temblando como cuerdas de arpa,

como telarañas al viento [...] (p. 187) ${ }^{10}$.

Le hurlement du chien, symboliquement gardien et guide du troupeau, serait une manière de prévenir du malheur à venir. Ce cri prédispose une ambiance de terreur généralisée, ressentie par tous et même par l'environnement. L’insistance de la musique traduit aussi un sentiment nostalgique relatif au dénouement tragique de l'épisode :

[...] para que usted sepa que allá, en la placita de nuestro pueblo, está su hijo Cornelio, Capitán del Ejército Peruano, quién - como me dice usted - ya la visitó en horas de la matanza y que ahora está llorando harto, bramando como un torillo herido, bien abrazado al cuerpo de su hermano Modesto... (p. 188).

Les rôles s'inversent : c'est au tour du militaire de crier sa douleur face à la mort d'un de ses proches. Le caractère absurde du dénouement, tenu en haleine jusqu'à la fin, ainsi que la morale suggérée, condamnent clairement les abus de la guerre durant laquelle des frères s'entretuent.

En conclusion, il semble clair que l'écriture reflète et même transcende la réalité vécue durant le conflit interne. Rendre compte de la situation à travers le regard et la voix d'un Andin permet d'entrevoir une autre réalité, celle mise en évidence par la Commission de la Vérité et de la Réconciliation depuis 2001. En effet, le conte dénonce les pratiques des forces armées et ignore, dans son développement, les sentiéristes, jusqu'alors uniques responsables de la violence commise. Ce témoignage rompt donc avec l'histoire officielle mais aussi, dans sa transcription littéraire, avec les stratégies narratives mises en œuvre. Loralité suggérée, caractéristique du conte et de la tradition andine, serait une manière de résister et d'informer ceux qui refusent de regarder vers cet autre monde.

Avec la contribution de Christine Pommiers lagrégée d'espagnol au lycée du Couserans à Saint-Girons en Ariègel pour la traduction des citations et de l'épigraphe.

(10) Je propose de donner au texte l'aspect que lui confère son écriture poétique. 